\title{
Oncology during the COVID-19 pandemic: challenges, dilemmas and the psychosocial impact on cancer patients (Review)
}

\author{
KONSTANTINOS TSAMAKIS ${ }^{1,2}$, MARIA GAVRIATOPOULOU ${ }^{3}$, DIMITRIOS SCHIZAS ${ }^{4}$, \\ ATHINA STRAVODIMOU ${ }^{5}$, AIKATERINI MOUGKOU ${ }^{6}$, DIMITRIOS TSIPTSIOS ${ }^{7}$, \\ VASILEIOS SIOULAS ${ }^{8}$, ELEFTHERIOS SPARTALIS $^{9}$, ATHANASIOS D. SIOULAS ${ }^{10}$, \\ CHARALAMPOS TSAMAKIS ${ }^{11}$, NIKOLAOS CHARALAMPAKIS ${ }^{12}$, CHRISTOPH MUELLER ${ }^{2,13}$, \\ DONNA ARYA ${ }^{14}$, PAUL ZAROGOULIDIS ${ }^{15}$, DEMETRIOS A. SPANDIDOS ${ }^{16}$, \\ MELETIOS A. DIMOPOULOS ${ }^{17}$, CHARALABOS PAPAGEORGIOU ${ }^{18}$ and EMMANOUIL RIZOS ${ }^{1}$ \\ ${ }^{1}$ Second Department of Psychiatry, University of Athens, ‘ATTIKON' University Hospital, 12462 Athens, Greece; \\ ${ }^{2}$ King's College London, Institute of Psychiatry, Psychology and Neuroscience, London SE5 8AF, UK; \\ ${ }^{3}$ Department of Therapeutics, Alexandra Hospital, National and Kapodistrian University of Athens, 11528 Athens; \\ ${ }^{4}$ First Department of Surgery, National and Kapodistrian University of Athens, Laikon General Hospital, 11527 Athens, \\ Greece; ${ }^{5}$ Department of Oncology, Centre Hospitalier Universitaire Vaudois, Lausanne University Hospital, \\ CH-1011 Lausanne, Switzerland; ${ }^{6}$ Paediatric Infectious Diseases Dept, Karolinska University Hospital, 17177 Stockholm, \\ Sweden; ${ }^{7}$ Department of Neurophysiology, South Tyneside and Sunderland NHS Foundation Trust, Sunderland SR47TP, UK; \\ ${ }^{8}$ Department of Gynaecologic Oncology MITERA Hospital, 15123 Athens; ${ }^{9}$ 2nd Department of Propaedeutic Surgery, \\ National and Kapodistrian University of Athens School of Medicine, 11527 Athens; ${ }^{10}$ Gastroenterology Department, \\ Hygeia Hospital, 15123 Athens, Greece; ${ }^{11}$ Department of Dermatology, Luton and Dunstable University Hospital, \\ Bedfordshire Hospitals NHS Foundation Trust, Luton LU4 0DZ, UK; ${ }^{12}$ Department of Medical Oncology, \\ Metaxa Cancer Hospital, Pireas, 18537 Athens, Greece; ${ }^{13}$ South London and Maudsley NHS Foundation Trust, \\ London SE5 8AZ; ${ }^{14}$ Thornford Park, Elysium Healthcare, Berkshire RG19 8ET, UK; ${ }^{15}$ 3rd Department of Surgery, \\ 'AHEPA' University Hospital, Aristotle University of Thessaloniki, Medical School, 54621 Thessaloniki; \\ ${ }^{16}$ Laboratory of Clinical Virology, School of Medicine, University of Crete, 71003 Heraklion; \\ ${ }^{17}$ Hematology and Medical Oncology, Department of Clinical Therapeutics, National and \\ Kapodistrian University of Athens, School of Medicine, 11527 Athens; ${ }^{18}$ First Department of \\ Psychiatry, University of Athens, 'EGINITION' Hospital, 11528 Athens, Greece
}

Received April 13, 2020; Accepted May 8, 2020

DOI: $10.3892 / \mathrm{ol} .2020 .11599$

\begin{abstract}
COVID-19 has caused unprecedented societal turmoil, triggering a rapid, still ongoing, transformation of healthcare provision on a global level. In this new landscape, it is highly important to acknowledge the challenges this pandemic poses on the care of the particularly vulnerable cancer patients and the subsequent psychosocial impact on them. We have outlined our clinical experience in managing patients with gastrointestinal, hematological, gynaecological, dermatological, neurological, thyroid, lung and paediatric
\end{abstract}

Correspondence to: Dr Konstantinos Tsamakis, Second Department of Psychiatry, University of Athens, 'ATTIKON' University Hospital, Rimini 1, Chaidari, 12462 Athens, Greece

E-mail: ktsamakis@gmail.com

Key words: COVID-19, pandemic, SARS-Cov-2, cancer, oncology, care, challenges, psychosocial impact, patients, telemedicine cancers in the COVID-19 era and have reviewed the emerging literature around barriers to care of oncology patients and how this crisis affects them. Moreover, evolving treatment strategies and novel ways of addressing the needs of oncology patients in the new context of the pandemic are discussed.

\section{Contents}

1. Introduction

2. Oncology care in general

3. Challenges in managing specific types of cancers

4. Conclusions

\section{Introduction}

Since December 2019 the COVID-19 pandemic has affected all aspects of our lives worldwide. This unparalleled health crisis (1), for which no healthcare system was fully prepared, has 
caused a tremendous strain in healthcare services (2), affecting directly and indirectly the course and treatment of many common illnesses (3). Oncology patients have been particularly affected, since they are regarded a highly vulnerable group in the current pandemic, due to their immunocompromised status caused by both cancer and various anticancer treatments $(4,5)$. Data from China and Italy show that the case fatality (defined as the number of deaths in COVID-19 positive patients, divided by the number of those tested positive) is significantly higher in patients with cancer than those without (6 vs. $2 \%)(6,7)$. In addition, two recent studies demonstrated that patients with cancer are at an increased risk of more severe infection and subsequent complications, particularly if surgery or chemotherapy are performed within the month preceding SARS-Cov-2, while an underlying cancer diagnosis is associated with an increased risk of death and/or intensive care unit (ICU) admission $(8,9)$.

Although the benefits of cancer therapies remain the same in the current crisis, the risks have increased substantially, warranting careful reconsideration of clinical practices (10). As such, the COVID-19 pandemic has significantly altered cancer patient management (11) as evidenced by local (12), national (13), European (14) and American (15) guidelines or recommendations that have been recently published.

The present review explores the challenges to Oncology care that these new changes generate, and the subsequent psychosocial impact this has on cancer patients.

\section{Oncology care in general}

New realities. In this new landscape, the cancer community is steering a difficult course amid several challenges: revisiting optimal standards of cancer care, facing persistently evolving shortages and acknowledging the paradoxical need to keep patients away from health care facilities. An ongoing challenge is balancing the risk of admission to the hospital with the possible risk of a SARS Cov 2 infection, which has an emotional impact not only on patients, but also on medical staff. Clinicians are under intense pressure and scrutiny during the COVID-19 pandemic, with oncology physicians particularly pressured, as they are facing several ethical dilemmas, as well as physical isolation from friends and family, increased risk of disease transmission, clinical challenges and competing demands (16).

Psychological impact on patients. Patients themselves have been deeply affected by the new realities; in particular by the knowledge of the higher risk of serious complications if infected by SARS-Cov-2 and the underlying fear of how the potential healthcare-capacity issues will interfere with the optimal treatment of their disease. COVID-19 can trigger anxiety and distress, which may be of increased intensity in vulnerable patient groups, such as the oncology patients. Cancer patients feel isolated and can even experience emotions of guilt if their family provides help with daily living activities, which can be opposed to the recommended social distancing (26). Quarantine, a common reality during the COVID-19 pandemic, may precipitate anxiety, irritability and depression, especially when implemented for prolonged periods (17). The social isolation and the need to 'stay at home' cause loneliness, which is associated with a higher risk of mortality in cancer patients $(18,19)$. It is well documented that perceived uncertainty increases individual emotional distress, and this, in turn, has negative effects on clinical outcomes in cancer patients (20-22).

Altering treatments and guidelines. Oncology societies and national authorities have been quick to issue guidelines on cancer care during the pandemic. The prioritization in the delivery of cancer therapies is strongly influenced by both the magnitude of potential treatment benefits and therapeutic intent, while patient-level factors such as age, comorbidities, and patient preferences have to be considered in the general framework (23). Strategies such as reducing the number of clinical visits, shortening radiotherapy fractionation, converting intravenous to oral systemic therapy, and even stopping or reducing the frequency of selected maintenance treatments have been implemented in most of the oncology clinics.

Addressing patients' needs. Addressing the concerns of patients poses another challenge to the oncology institutes. During this crisis, ensuring the well-being of the patients and caregivers, providing a safe environment and effective and compassionate care are more important than ever. Patients have been encouraged to explore creative ways to cope with stress, including physical activity, a balanced diet, adequate sleep, mindful-based strategies, avoiding overload of information and social connectedness. Additional support through mental health services should be available if the aforementioned measures are not sufficient. Virtual community and patient support groups can be excellent sources of encouragement and support, both for oncology patients and their caregivers (24).

Communication, remote care and e-Health. In this turmoil, better and efficient communication between oncologists and their patients is of utmost priority. Telemedicine has been used by oncologists and patients with a positive experience in both groups $(25,26)$. Although its effectiveness at reducing the spread of infection and improving outcomes is not yet proven (27), it increases access to care. Interacting with patients via telemedicine and providing useful advice both on somatic and mental matters could help avoid non-essential admissions to hospital (28). Telemedicine is also used by onco-psychologists and onco-psychiatrists to guarantee psychological support for individual patients, while limiting visits to the cancer institute.

In response to cancer patients' increased need of efficient and helpful communication, also highlighted by cancer patient groups, the websites of most cancer institutes have added a regularly updated question-and-answer COVID-19 section, whilst social media campaigns and press releases offer additional channels to reach out to patients. In particular, the use of social media could help overcome certain barriers posed by the COVID-19 pandemic, as previous experience in cancer patients shows that social media use had a positive impact on psychosocial health outcomes, e.g., larger social support networks were associated with better prognosis after breast cancer treatment. More specifically, a study by Shim et al found that those who disclosed personal insights about breast cancer in an online support group experienced higher health self-efficacy and emotional and functional well-being (29), while users of a Twitter breast cancer support group also reported decreased anxiety after tweet chats (30). 
Additional virtual care tools, such as webinars, can also be used to support patients. Although synchronous (e.g., 'Zoom groups') and asynchronous (e.g., Facebook) cancer specific groups may be viable options, it is essential to consider issues of privacy and credibility of information shared. The rapid need for implementation of virtual care during the COVID-19 pandemic will necessitate further research to help improve our understanding of e-Health effectiveness in cancer patients.

\section{Challenges in managing specific types of cancers}

Haematological cancer patients. COVID-19 is expected to be a devastating infection in many patients with solid tumours and hematologic malignancies due to older age, comorbidities and immunocompromised status and therefore should be managed appropriately without jeopardizing the curative chance of these patients. In the COVID-19 oncology era, difficult clinical decisions regarding delay or initiation of chemotherapy need to be carefully evaluated. Adjuvant therapy with curative objective should proceed during the pandemic, while for metastatic disease, any delays might lead to further progression and ineligibility for further treatment (31). The American Society of Clinical Oncology recommends rescheduling routine follow-up visits of patients that are not currently receiving treatment. Home collection of lab samples should be considered to avoid additional visits to the clinic (32). If possible, it is considered reasonable to delay highly immunosuppressive treatment strategies such as allogeneic or autologous stem cell transplantation (33). Chemotherapy protocols are being revised in order to minimize frequency of visits and depth of immunosuppression. Oral regimens should be preferred, especially for elderly and frail patients. Watchful waiting should be considered for patients with asymptomatic and slow progressions.

Another challenge is that patients under cytoreductive treatment or immunotherapy, as well as patients with lung cancer or metastatic lesions, might present with symptoms similar to COVID-19, either due to other infectious diseases or to the underlying malignancy. Therefore, it is of utmost importance to educate cancer patients accordingly and evaluate them in the most appropriate way. Patients need to be informed regarding the symptoms of COVID-19, trained in proper hand washing hygiene and guided on using personal protective equipment whenever they go out in public places or the hospital.

Neurological cancer patients. Apart from social isolation and physical distancing resulting in anxiety and distress, the quality of life of patients with brain tumors, who often have to travel to hospitals in endemic areas, is significantly affected by the fear of facing this pandemic with an immune system that is significantly weakened by their disease, steroids, radiotherapy or chemotherapy (34). Neuro-oncologists and neurosurgeons not only face the ethical dilemma of treating their immunocompromised patients at the same hospital as COVID-19 patients, but also realize that they cannot effectively deliver their services, as most neurosurgical oncology cases demand ICU admission in the early postoperative period. This is often impossible, as ICUs prioritize and reserve capacity for COVID-19 patients. In a highly endemic area, such as Lombardy, Italy, these difficulties have been effectively addressed by designating a regional dedicated COVID-19 free neuro-oncology center where all patients before admission undergo 'COVID-19 screening' with body temperature, $\mathrm{O}_{2}$ saturation, C-reactive protein, SGOT, SGPT levels, complete cell blood count, chest radiography, pharyngeal SARS-CoV-2 swab and a thorough interview. This has resulted in the treatment of a high volume of COVID-19 negative patients and high levels of patient satisfaction (35)

In places where COVID-19 free centers are not available, current management is based on triage. Telephone or video visits are utilized in order to assess routine follow-ups and management of asymptomatic benign or low-grade tumors is postponed until a safer time, whereas malignant tumors and emergency cases are treated as in the pre-COVID-19 era. In order to avoid occupational exposure all patients requiring surgery should undergo 'COVID-19 screening'. If they are COVID-19 positive they need to be transferred to dedicated COVID-19 hospitals or wards where appropriate personal protective equipment for health care professionals, as well as negative pressure operating rooms (ORs) and corresponding ICU are available (36).

For patients undergoing chemotherapy conservative dosing is recommended in order to avoid immunosuppression. If viral symptoms occur patients should be immediately tested for SARS-CoV-2 and in case of positivity chemotherapy should be held until the patient fully recovers from the infection. Risks and benefits of therapy should then be re-evaluated (37), however, in some centers two negative SARS-CoV-2 tests are required before re-initiation of chemotherapy. Finally, concerning radiation therapy, strict institutional policies are required in order to guarantee patient and health care workers' safety, as daily hospital visits are needed. In patients with suspected or confirmed SARS-CoV-2 infection radiotherapy can be either withheld, or continue as long as their disease is mild and the radiation facility has measures in place that prevent potential spread to other patients and staff. On the other hand, in cases with significant viral symptoms, treatment should be halted and resumed only after patient recovery (38).

Gastrointestinal (GI) cancer patients. Gastrointestinal (GI) cancer is a very serious condition and the recent COVID-19 outbreak is an additional obstacle in the management of such patients. Of note, $1 \%$ of all patients who contracted COVID-19 had a history of cancer. In addition, a previous history of cancer has been associated with an increased risk of morbidity and mortality following SARS-CoV-2 infection, which has caused considerable distress among GI cancer patients (39).

The fear of spread of this novel disease, alongside the effort to preserve hospital resources, including ventilators, personal protective equipment (PPE), critical care resources, and blood products, has led many institutions to limit or postpone elective cancer procedures and cancel outpatient clinics. Lockdown and disruptions in transportation have made access to diagnostic procedures, such as biopsies difficult. Consequently cancer patients are experiencing an unexpected delay in diagnosis, staging and cancer therapy, causing a further psychological burden (28) which is aggravated by the 'combined' fear of both cancer and COVID-19 (40). Depression rates appear to have increased in GI cancer patients, as the relationship with their treating clinician has become more limited due to quarantine and social distancing and support from relatives is missing (41). 
The new reality has forced the medical community to prioritize and explore alternative, non-surgical therapeutic approaches for GI cancer patients. In advanced staged GI cancer patients in particular, initiation or continuing of chemotherapy has been implemented, although the induced immunosuppression raises the risk for increased morbidity and death in the event of a SARS-CoV-2 infection (42). On the other hand, delays in treatment for GI cancer patients could result in unfavorable clinical outcomes, as a number of elective surgeries for GI cancer become urgent as time passes (43). After all, a 'reasonable' time-frame should be maintained for GI cancer patients, if the medical community does not want to report such patients as the 'occult pandemic victims'.

Lung cancer patients. COVID-19 pandemic has affected the management of patients with lung cancer rather significantly. Risk factors for COVID-19 related complications in this patient group include older age, significant cardiovascular and respiratory co-morbidities, smoking-related lung damage, poor nutritional status and treatment related immunosuppression $(8,44)$. One of the most critical issues of managing lung cancer patients during this pandemic, is the overlap between the radiological manifestations and especially the CT findings of COVID-19 induced pneumonia, with those that are often found upon disease progression, or onset of concomitant pneumonia due to overlapping opportunistic infections, or immune-checkpoint related pneumonitis $(45,46)$. As a result, distinguishing lung cancer progression or a drug-related complication from potential COVID-19 infection is a great challenge for clinicians, especially since different therapeutic approaches (47) and strategies are required on each occasion. Thus, the management of lung cancer patients should undoubtedly involve increased attention to their clinical and radiological pulmonary signs.

In this context, and considering the high transmissibility rate of SARS-Cov-2, standard-of-care treatment regimens have been adapted for lung cancer patients, mainly in order to minimize the number of hospital visits and hospitalization and to prevent anticancer treatment-induced complications. Furthermore, oncology canters have implemented several strategies, also followed on other types of cancer, such as converting intravenous treatments to oral regimens whenever possible, switching cytotoxic chemotherapy to less toxic treatments, pausing therapies once the disease has become stable and replacing oncological surgery with stereotaxic radiotherapy in early stage disease (IA-IB).

Patients with malignant melanoma. The COVID-19 pandemic has posed new challenges in managing patients with malignant melanoma, a less common but far more dangerous type of skin cancer, because of its ability to metastasize to other organs, if not treated at an early stage (48). During this novel pandemic, scheduled follow-up appointments for melanoma patients have been cancelled or replaced by telephone consultations. Frequently there are temporary delays in organizing surgical procedures for removal of suspicious moles and imaging tests, including staging $\mathrm{CT}$ and MRI scans. This further increases the uncertainty patients and their caregivers are already experiencing.

Many of the melanoma patients, especially those with advanced disease, are being treated with targeted therapy or immunotherapy in an adjuvant or palliative setting (49). Considering that these cancer related treatments may cause immunosuppression, patients' anxiety increases further as they feel more vulnerable to contracting COVID-19. Experience from patient support groups shows that many melanoma survivors express stress and feelings of helplessness due to the new pandemic. This is further aggravated by the fact that melanoma support groups are no longer able to meet (50). Amid this overwhelming pandemic though, community support groups consisting of volunteers are being set up, in order to support isolated melanoma patients (51).

Gynaecological cancers. As with other malignancies, the care of women suffering from gynaecological cancers has been severely affected by the COVID-19 pandemic. Several modalities are used in the treatment of gynaecological cancers: surgery, radiation and intravenous systemic therapy, including chemotherapy, targeted therapies and immunotherapy. From the surgical point of view, the availability of OR space and personnel for prolonged operations, blood bank resources and ICU beds may directly impact the decision of whether to proceed with an operable case, weighed against the oncological safety of its delay $(52,53)$. Even the theoretical pneumoperitoneum-related risks of minimally invasive surgery in the COVID-19 context should be carefully weighed against the higher morbidity linked to laparotomy, along with the latter's need for prolonged hospitalization $(52,54)$ As far as chemotherapy and other anti-cancer treatments are concerned, the possible immunocompromising of patients may increase their susceptibility to viral infections and severe clinical manifestations $(52,55)$. Of note, experience of oncology patients in Wuhan lends support to the above $(8,44)$. Lastly, barriers pertaining to the access to diagnosis and treatment for patients with symptoms indicative of gynaecological cancer should not be underestimated.

To maximize the quality of gynaecological cancer care, alongside the safety of the patients, their families and health professionals, all the major societies in the field released guidelines that, in general, focus on: 'less surgery, less hospital visits and more protective measures against the COVID-19 spread' $(52,53,55,56)$. The application of these recommendations to current clinical practice is highly dependent on the local/regional pandemic statistics, infrastructure of health services and individual patient's needs. Importantly, the formulation of the treatment plan should follow an open and meticulous discussion with the patient, extending from the pre-pandemic standard of care to the oncologic implications of the emerging alternatives and the risks of SARS-CoV-2 infection.

Thyroid cancer. Despite progress in thyroid cancer diagnosis and treatment resulting in increased operative options and improved surveillance strategies, it should be kept in mind that every hospital visit, diagnostic intervention or operation, exposes patients and health care providers to the risk of contracting COVID-19. The American College of Surgeons (ACS) (57) issued a statement calling for a thoughtful review of all scheduled elective procedures with a plan to minimize, postpone, or cancel electively scheduled operations, endoscopies, or other invasive procedures, until there is confidence that the health care infrastructure can support a potentially rapid and overwhelming uptick in critical patient care needs. 
It is highly probable that cancer morbidity or even mortality will rise, not only because of the direct implications of SARS-CoV-2 infections, but also because of deviations from usual cancer care pathways. Previous experience shows that, during pandemics, healthcare systems implement a triage system that could potentially deny critical care treatment to some seriously ill patients (58).

COVID-19 has entered mainstream consciousness and disrupted normal flow of thyroid surgeries. Furthermore, it increased patient's anxiety regarding diagnosis and prompt treatment. Elective interventions and non-emergency operations are deferred (59) and further new challenges have arisen. For instance, a mild airway compression from large goiters is subacute or chronic, and does not cause symptoms (60); but what if such a patient requires immediate intubation due to acute COVID-19-induced respiratory distress? This could result in significant and probably fatal airway compromise and subsequently many patients with large goiters are very concerned about their postponed surgeries.

In addition, most differentiated (DTC) and medullary thyroid cancers (MTC) are slow-growing tumors that do not cause significant short-term morbidity, but there is a subset of more aggressive cancers that progress more rapidly. Therefore, clinical correlation with rate of progression, size, invasiveness of surrounding structures, and proximity to critical structures is recommended in the COVID-19 context (61). In patients living with such types of asymptomatic cancer, the disease might progress in the absence of treatment. This might lead to metastatic disease or even death, or make more complex operations necessary, with increased complication rates (i.e., MTC or some rare invasive types of DTC) (62-64).

Paediatric oncology. Although attention on the consequences of the COVID-19 pandemic has mainly focused on adult patients, children, especially those in high risk groups such as the immunocompromised, are also affected mentally and physically. Bouffet et al (65) highlighted the need for urgent preparedness to protect the paediatric oncology population by sharing knowledge and experience on the impact of the pandemic on these patients. An international paediatric haematology/oncology group provided data on COVID-19 incidence in almost 10,000 children treated with chemotherapy or intensive immunosuppression. Of 200 symptomatic children tested, only 8 patients were found to be positive for the SARS-CoV-2, leading to the proposition that a SARS-CoV-2 infection might be expressed as mild disease in children with cancer (66).

However, the indirect impact of the COVID-19 pandemic on children with cancer and their families should not be overlooked. A recent study in cancer patients in Milan, which involved adolescents among other young participants, revealed that a large proportion of patients with cancer were worried and felt personally at risk of severe complications, with their parents' concern for them as an additional burden (67). In addition, the reduction of resources, in both materials and staff, has resulted in decreased access to routine care. An overwhelmed healthcare system could lead to limited support for the multiple needs of this vulnerable population, such as intravenous treatments at home, palliative care and low-threshold access to hospital.

Moreover, paediatric oncology patients face an increased risk of infections (68). The single most effective interven- tion to reduce the transmission of infectious agents is good hand hygiene. Unfortunately low adherence to hand hygiene protocols can occur in paediatric oncology departments as demonstrated by Kouni et al (69) in a study where only $25.7 \%$ of staff practiced appropriate hand hygiene measures while caring for children with cancer. However, since the emergence of SARS-CoV-2 there has been an increasing investment in capacity building, education and training in hand hygiene measures. This might be a positive outcome of the pandemic after all, with lessons on the importance of prevention of infections learned both by healthcare staff and the general public.

\section{Conclusions}

Oncology patients deserve particular attention during the current pandemic as they are immunocompromised and vulnerable to severe of outcomes of a SARS-CoV-2 infection, due to both the underlying malignancy and various anticancer treatments.

Cancer therapies are of proven benefit, but in light of the serious consequences of potential concurrent SARS-CoV-2 infection, risk-to-benefit considerations are becoming increasingly important. For each individual patient their disease status, age, frailty, and comorbidities (10) should be considered, as well as the potential of alternative strategies of care delivery. When possible, oral regimens should be preferred, and regimen administration intervals could be redefined, while postponing treatments or other invasive investigations could be considered for patients with asymptomatic and slow progressing cancers.

Telemedicine is increasingly being used by oncologists and patients with largely positive experiences for both groups. This process needs to be accelerated, increasing accessibility and quality of the interaction and data gathered. Furthermore, the threshold for patients with signs or symptoms of potential underlying malignancies accessing diagnosis and treatment should be kept as low as possible. The psychological impact of not only the cancer, but also the increased social distancing and shielding measures many of the patients face, requires consideration. Patient support groups online and access to supportive and more formal psychological help need to be planned and be accessible. Lastly, the current situation of the pandemic will force clinicians to make difficult decisions; however, if decisions are made thoughtfully, transparently and in collaboration with patients and carers (70) confidence in the healthcare system will remain high.

\section{Acknowledgements}

Not applicable.

\section{Funding}

No funding was received.

\section{Availability of data and materials}

Not applicable. 


\section{Authors' contributions}

KT wrote the original draft, edited and critically revised the manuscript. MG, DS, AS, AM, DT, ES, VS, ADS, CT, NC and PZ contributed substantially in the writing of the manuscript, critically revised and edited the manuscript. ER, DA, MAD, CP, DAS and CM critically revised and edited the manuscript. All authors substantially contributed to the conception, writing and revision of the work and approved the final content of the manuscript.

\section{Ethics approval and consent to participate}

Not applicable.

\section{Consent for publication}

Not applicable.

\section{Competing interests}

DAS is the Editor-in-Chief for the journal, but had no personal involvement in the reviewing process, or any influence in terms of adjudicating on the final decision, for this article. The other authors declare that they have no competing interests.

\section{References}

1. Ahmad A, Mueller C and Tsamakis K: Covid-19 pandemic: A public and global mental health opportunity for social transformation? BMJ 369: $\mathrm{m} 1383,2020$.

2. Docea AO, Tsatsakis A, Albulescu D, Cristea O, Zlatian O, Vinceti M, Moschos SA, Tsoukalas D, Goumenou M, Drakoulis N, et al: A new threat from an old enemy: Re-emergence of coronavirus (Review). Int J Mol Med 45: 1631-1643, 2020.

3. Tsamakis K, Triantafyllis A, Tsiptsios D, Spartalis E, Mueller C, Tsamakis C, Chaidou S, Spandidos DA, Fotis L, Economou M and Rizos E: COVID 19 related stress exacerbates common physical and mental pathologies and affects treatment (Review). Exp Ther Med (In Press).

4. Kamboj M and Sepkowitz KA: Nosocomial infections in patients with cancer. Lancet Oncol 10: 589-597, 2009.

5. Schrag D, Hershman DL and Basch E: Oncology practice during the COVID-19 pandemic. JAMA: Apr 13, 2020 (Epub ahead of print).

6. Onder G, Rezza G and Brusaferro S: Case-fatality rate and characteristics of patients dying in relation to COVID-19 in Italy. JAMA: Mar 23, 2020 (Epub ahead of print).

7. $\mathrm{Wu} \mathrm{Z}$ and McGoogan JM: characteristics of and important lessons from the coronavirus disease 2019 (COVID-19) outbreak in China: Summary of a report of 72,314 cases from the Chinese Center for Disease Control and Prevention. JAMA: Feb 24, 2020 (Epub ahead of print).

8. Liang W, Guan W, Chen R, Wang W, Li J, Xu K, Li C, Ai Q, $\mathrm{Lu}$ W, Liang H, et al: Cancer patients in SARS-CoV-2 infection: A nationwide analysis in China. Lancet Oncol 21: 335-337, 2020

9. Zhang L, Zhu F, Xie L, Wang C, Wang J, Chen R, Jia P, Guan HQ, Peng L, Chen Y, et al: Clinical characteristics of COVID-19-infected cancer patients: a retrospective case study in three hospitals within Wuhan, China. Ann Oncol: Mar 26, 2020 (Epub ahead of print).

10. National Comprehensive Cancer Network (NCCN): Principles for management of colorectal cancer patients during the COVID-19 pandemic. https://www.ncen.org/covid-19/pdf/ColorectalCOVID-19. pdf. Accessed January 5, 2020.

11. Al-Quteimat OM and Amer AM: The impact of the COVID-19 pandemic on cancer patients. Am J Clin Oncol: Apr 16, 2020 (Epub ahead of print).

12. The Royal College of Radiologists: Neuro-oncology treatment guidance during COVID-19 pandemic. https://www.rer. ac.uk/sites/default/files/neuro-oncology-treatment-covid-19.pdf?utm medium=email\&utm_source=transaction. Accessed March 27, 2020 .
13. British Neuro-Oncology Society (BNOS): Adult Neuro-Oncology Service Provision during COVID-19 outbreak. https://www.bnos. org.uk/wp-content/uploads/2020/03/Adult-neuro-oncology-service -provision-during-COVID-outbreak_SBNS-BNOS.pdf. Accessed March 19, 2020.

14. European Association of Neuro-Oncology (EANO): COVID-19 and Neuro-oncology: considerations for daily care of brain tumor patients. EANO, Vienna, 2020. https://www.eano.eu/.

15. Ramakrishna R, Zadeh G, Sheehan JP and Aghi MK: Inpatient and outpatient case prioritization for patients with neuro-oncologic disease amid the COVID-19 pandemic: general guidance for neuro-oncology practitioners from the AANS/CNS Tumor Section and Society for Neuro-Oncology. J Neurooncol: Apr 9, 2020 (Epub ahead of print).

16. Tsamakis K, Rizos E, Manolis JA, Chaidou S, Kympouropoulos S, Spartalis E, Spandidos DA, Tsiptsios D and Triantafyllis AS: [Comment] COVID-19 pandemic and its impact on mental health of healthcare professionals. Exp Ther Med 19: 3451-3453, 2020.

17. Brooks SK, Webster RK, Smith LE, Woodland L, Wessely S, Greenberg N and Rubin GJ: The psychological impact of quarantine and how to reduce it: Rapid review of the evidence. Lancet 395: 912-920, 2020.

18. D'Ippolito S, Ambrosini E, Shams M, Calì G and Pastorelli D: The effect of loneliness on cancer mortality. Ann Oncol 28: vi82, 2017.

19. Hill EM and Hamm A: Intolerance of uncertainty, social support, and loneliness in relation to anxiety and depressive symptoms among women diagnosed with ovarian cancer. Psychooncology 28: 553-560, 2019.

20. Bortolato B, Hyphantis TN, Valpione S, Perini G, Maes M, Morris G, Kubera M, Köhler CA, Fernandes BS, Stubbs B, et al: Depression in cancer: The many biobehavioral pathways driving tumor progression. Cancer Treat Rev 52: 58-70, 2017.

21. Reis JC, Antoni MH and Travado L: Emotional distress, brain functioning, and biobehavioral processes in cancer patients: A neuroimaging review and future directions. CNS Spectr 25: 79-100, 2020.

22. Papanastasiou A, Seliniotaki T, Rizos E, Kampoli K, Ntavatzikos A, Arkadopoulos N, Tsionou C, Spandidos DA and Koumarianou A: Role of stress, age and adjuvant therapy in the cognitive function of patients with breast cancer. Oncol Lett 18: 507-517, 2019.

23. Hanna TP, Evans GA and Booth CM: Cancer, COVID-19 and the precautionary principle: Prioritizing treatment during a global pandemic. Nat Rev Clin Oncol 17: 268-270, 2020.

24. Centers for Disease Control and Prevention (CDC): Stress and Coping. Coronavirus Disease 2019 (COVID-19). https://www. cdc.gov/coronavirus/2019-ncov/daily-life-coping/managingstress-anxiety.html. Accessed April 28, 2020.

25. Gondal H, Choquette H, Abbas T, Le D, Chalchal HI, Iqbal N and Ahmed S: Patients and physicians' satisfaction with telemedicine (TM) in cancer care and factors that correlate with a positive patient's experience. Ann Oncol 30: v730, 2019.

26. Sirintrapun SJ and Lopez AM: Telemedicine in cancer care. Am Soc Clin Oncol Educ Book 38: 540-545, 2018.

27. Pham C and Badowski ME: The role of telemedicine in infectious diseases. EMJ Innov: Mar 26, 2019 (Epub ahead of print).

28. Cinar P, Kubal T, Freifeld A, Mishra A, Shulman L, Bachman J, Fonseca R, Uronis H, Klemanski D, Slusser K, et al: Safety at the time of the COVID-19 pandemic: How to keep our oncology patients and healthcare workers safe. J Natl Compr Canc Netw: Apr 15, 2020 (Epub ahead of print).

29. Attai DJ, Cowher MS, Al-Hamadani M, Schoger JM, Staley AC and Landercasper J: Twitter social media is an effective tool for breast cancer patient education and support: Patient-reported outcomes by survey. J Med Internet Res 17: e188, 2015.

30. Shim M, Cappella JN and Han JY: How does insightful and emotional disclosure bring potential health benefits?: Study based on online support groups for women with breast cancer. J Commun 61: 432-464, 2011

31. National Comprehensive Cancer Network (NCCN): NCCN Best Practices Committee Infusion Efficiency Workgroup. Toolkit: Providing Oncology Treatments in the Outpatient Setting. https://www.nccn.org/about/news/ebulletin/pdf/ NCCN\%200P\%20Toolkit\%20Final.pdf. Accessed August 2019.

32. American Society of Clinical Oncology (ASCO): COVID-19 Provider \& Practice Information. https://www.asco.org/ asco-coronavirus-information/provider-practice-preparedness -covid-19. Acessed April 28, 2020. 
33. American Society of Hematology: COVID-19 Resources. https:// www.hematology.org/covid-19\#faq. Accessed April 28, 2020.

34. Finocchiaro G: Milan 2020: COVID-19, neuro-oncology and much more. J Neurooncol: Apr 13, 2020 (Epub ahead of print).

35. Perin A, Servadei F and DiMeco F; 'Hub and Spoke' Lombardy Neurosurgery Group: May we deliver neuro-oncology in difficult times (e.g. COVID-19)? J Neurooncol: Apr 10, 2020 (Epub ahead of print).

36. Hu YJ, Zhang $\mathbf{J}$ and Chen Z: Experiences of practicing surgical neuro-oncology during the COVID-19 pandemic. J Neurooncol: Apr 10, 2020 (Epub ahead of print)

37. Mohile NA, Blakeley JO, Gatson NTN, Hottinger AF, Lassman AB, Ney DE, Olar A, Schiff D, Shih HA, Strowd R, et al: Urgent considerations for the neuro-oncologic treatment of patients with gliomas during the COVID-19 pandemic. Neuro Oncol: Apr 11, 2020 (Epub ahead of print).

38. Chen W, Su XY, Wang VJ, Wang EC, Xu R, Zhong S, Sun G and Xia X: Novel Coronavirus International Public Health Emergency: Guidance on Radiation Oncology Facility Operation. Adv Radiat Oncol: Apr 1, 2020 (Epub ahead of print).

39. Pellino G and Spinelli A: How COVID-19 outbreak is impacting colorectal cancer patients in Italy: a long shadow beyond infection. Dis Colon Rectum: Mar 17, 2020 (Epub ahead of print).

40. Barbieri L, Talavera Urquijo E, Parise P, Nilsson M, Reynolds JV and Rosati R: Esophageal oncologic surgery in SARS-CoV-2 (COVID-19) emergency. Dis Esophagus: Apr 23, 2020 (Epub ahead of print).

41. Scalea JR: The distancing of surgeon from patient in the era of COVID-19: Bring on the innovation. Ann Surg: April 13, 2020 (Epub ahead of print).

42. Qadan M, Hong TS, Tanabe KK, Ryan DP and Lillemoe KD: A multidisciplinary team approach for triage of elective cancer surgery at the Massachusetts General Hospital during the novel Coronavirus COVID-19 outbreak. Ann Surg: April 13, 2020 (Epub ahead of print).

43. Yu GY, Lou Z and Zhang W: Several suggestions of operation for colorectal cancer under the outbreak of coronavirus disease 2019 in China. Zhonghua Wei Chang Wai Ke Za Zhi 23: 208-211, 2020 (In Chinese).

44. Yu J, Ouyang W, Chua MLK and Xie C: SARS-CoV-2 transmission in patients with cancer at a Tertiary Care Hospital in Wuhan, China. JAMA Oncol: Mar 25, 2020 (Epub ahead of print).

45. Bernheim A, Mei X, Huang M, Yang Y, Fayad ZA, Zhang N, Diao K, Lin B, Zhu X, Li K, et al: Chest CT findings in coronavirus disease-19 (COVID-19): Relationship to duration of infection. Radiology 200463: 200463, 2020.

46. Remon J, Passiglia F, Ahn MJ, Barlesi F, Forde PM, Garon EB, Gettinger S, Goldberg SB, Herbst RS, Horn L, et al: Immune checkpoint inhibitors in thoracic malignancies: review of the existing evidence by an IASLC expert panel and recommendations. J Thorac Oncol: Mar 14, 2020 (Epub ahead of print).

47. Delaunay M, Prévot G, Collot S, Guilleminault L, Didier A and Mazières J: Management of pulmonary toxicity associated with immune checkpoint inhibitors. Eur Respir Rev 28: 190012, 2019.

48. Abbas O, Miller DD and Bhawan J: Cutaneous malignant melanoma: Update on diagnostic and prognostic biomarkers. Am J Dermatopathol 36: 363-379, 2014.

49. Conforti C, Giuffrida R, Di Meo N and Zalaudek I: Management of advanced melanoma in the COVID-19 era. Dermatol Ther: Apr 22, 2020 (Epub ahead of print).

50. AIM at Melanoma Foundation: Melanoma Survivorship In the Age of COVID-19. https://www.youtube.com/ watch?v=tulUC9fG09E. Accessed Apri 23, 2020.

51. Melanoma UK: Melanoma UK COVID-19 Community Support Group. https://www.melanomauk.org.uk/melanoma-uk-covid19-community-support-group.

52. Ramirez PT, Chiva L, Eriksson AGZ, Frumovitz M, Fagotti A, Gonzalez Martin A, Jhingran A and Pareja R: COVID-19 global pandemic: Options for management of gynecologic gancers. Int J Gynecol Cancer: doi: 10.1136/ijgc-2020-001419.

53. Society for Gynecologic Oncology (SGO): Surgical considerations for gynecologic oncologists during the COVID-19 pandemic. https://www.sgo.org/clinical-practice/management/. Accessed March 27, 2020.

54. AAGL-Elevating Gynecologic Surgery: COVID-19: Joint Statement on Minimally Invasive Gynecologic Surgery. https:// www.aagl.org/news/covid-19-joint-statement-on-minimally-inva sive-gynecologic-surgery/. Accessed March 27, 2020.
55. Pothuri B, Secord AA, Armstrong DK, Chan J, Fader AN, Huh W, Kesterson J, Liu JF, Moore K, Westin SN and Naumann RW: Anti-cancer therapy and clinical trial considerations for gynecologic oncology patients during the COVID-19 pandemic crisis. Gynecol Oncol (In Press)

56. European Society of Gynaecological Oncology (ESGO): ESGO Covid-19 Updates. https://www.esgo.org/esgo-covid-19-communication/.

57. American College of Surgeons: COVID-19: Guidance for Triage of Non-Emergent Surgical Procedures. https://www.facs.org/ covid-19/clinical-guidance/triage. Accessed March 17, 2020.

58. Downar J and Seccareccia D; Associated Medical Services Inc. Educational Fellows in Care at the End of Life: Palliating a pandemic: 'all patients must be cared for'. J Pain Symptom Manage 39: 291-295, 2010.

59. Salako O, Okunade K, Allsop M, Habeebu M, Toye M, Oluyede G, Fagbenro G and Salako B: Upheaval in cancer care during the COVID-19 outbreak. Ecancermedicalscience 14: ed97, 2020.

60. Chen AY: Ethical Framework for Head and Neck Endocrine Surgery in the COVID 19 pandemic. Authorea: doi:10.22541/ au.158775702.25904845.

61. Givi B, Schiff BA, Chinn SB, Clayburgh D, Iyer NG, Jalisi S, Moore MG, Nathan CA, Orloff LA, O'Neill JP, et al: Safety Recommendations for Evaluation and Surgery of the Head and Neck During the COVID-19 Pandemic. JAMA Otolaryngol Neck Surg: Mar 31, 2020 (Epub ahead of print).

62. Spartalis E, Moris D and Tomos P: Sternal metastasis as first presentation of a well-differentiated papillary thyroid carcinoma. Surgery 162: 1336-1337, 2017.

63. Spartalis ED, Karatzas T, Charalampoudis P, Vergadis C and Dimitroulis D: Neglected papillary thyroid carcinoma seven years after initial diagnosis. Case Rep Oncol Med 2013: 148973, 2013.

64. Damaskos C, Garmpis N, Valsami S, Spartalis E, Antoniou EA, Tomos P, Karamaroudis S, Zoumpou T, Pergialiotis V, Stergios K, et al: Histone deacetylase inhibitors: A novel therapeutic weapon against medullary thyroid cancer? Anticancer Res 36: 5019-5024, 2016

65. BouffetE,Challinor J,Sullivan M,Biondi A, Rodriguez-GalindoC and Pritchard-Jones K: Early advice on managing children with cancer during the COVID-19 pandemic and a call for sharing experiences. Pediatr Blood Cancer: Apr 2, 2020 (Epub ahead of print).

66. Hrusak O, Kalina T, Wolf J, Balduzzi A, Provenzi M, Rizzari C, Rives S, Del Pozo Carlavilla M, Alonso MEV, Domínguez-Pinilla $\mathrm{N}$, et al: Flash survey on severe acute respiratory syndrome coronavirus-2 infections in paediatric patients on anticancer treatment. Eur J Cancer 132: 11-16, 2020.

67. Casanova M, Pagani Bagliacca E, Silva M, Patriarca C, Veneroni L, Clerici CA, Spreafico F, Luksch R, Terenziani M, Meazza C, et al: How young patients with cancer perceive the COVID-19 (coronavirus) epidemic in Milan, Italy: Is there room for other fears? Pediatr Blood Cancer: Apr 2, 2020 (Epub ahead of print).

68. Simon A, Ammann RA, Bode U, Fleischhack G, Wenchel HM, Schwamborn D, Gravou C, Schlegel PG, Rutkowski S, Dannenberg C, et al: Healthcare-associated infections in pediatric cancer patients: Results of a prospective surveillance study from university hospitals in Germany and Switzerland. BMC Infect Dis 8: 70, 2008.

69. Kouni S, Kourlaba G, Mougkou K, Maroudi S, Chavela B, Nteli C, Lourida A, Spyridis N, Zaoutis T and Coffin S: Assessment of hand hygiene resources and practices at the 2 children's hospitals in Greece. Pediatr Infect Dis J 33: e247-e251, 2014.

70. Dietz JR, Moran MS, Isakoff SJ, Kurtzman SH, Willey SC, Burstein HJ, Bleicher RJ, Lyons JA, Sarantou T, Baron PL, et al: Recommendations for prioritization, treatment, and triage of breast cancer patients during the COVID-19 pandemic. the COVID-19 pandemic breast cancer consortium. Breast Cancer Res Treat: Apr 24, 2020 (Epub ahead of print).

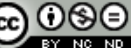

This work is licensed under a Creative Commons Attribution-NonCommercial-NoDerivatives 4.0 International (CC BY-NC-ND 4.0) License. 